\title{
Le couvre-feu et la lutte contre la propagation de la Covid-19 dans la ville de Lubumbashi
}

\author{
Par MUMBA KAKUDJI Martial* et MFUAMBA MULUMBA Isidore**
}

Après une longue période de confinement et d'état d'urgence sanitaire en République Démocratique du Congo, il a été instauré un couvre-feu devant servir à éviter la propagation de la deuxième vague de Covid-19. Observant les mesures d'exécution de ce couvre-feu et la pratique dans la ville de Lubumbashi, on a tendance de conclure que ce dernier n'a pas servi seulement à lutter contre la propagation du Coronavirus. Mais aussi, c'est un moyen nécessaire pour le renforcement de la sécurité et contrôle dans le pays d'une part et il est aussi une porte ouverte aux violations des droits de l'homme par les services de sécurit, d'autre part. Cette réflexion analyse l'impact du couvre-feu dans la ville de Lubumbashi en trois axes : le plan sanitaire; le plan sécuritaire et le plan des droits de l'homme.

Mots clés: couvre-feu, covid-19, droits de l'homme, police administrative, sécurité.

$$
* * *
$$

\section{Introduction}

Depuis le vendredi 18 décembre 2020, un couvre-feu national est décrété en République Démocratique du Congo à partir de 21 heures à 5 heures du matin [repoussé de 22 heures à 4 heures en avril $2021^{1}$ ]. La raison majeure et apparente de l'instauration de ce couvre-feu est de « lutter contre la propagation du Coronavirus dont le nombre de cas est reparti à la hausse depuis le mois de novembre ${ }^{2}$ ». L'observation mène à conclure que cette raison non seulement qu'elle est difficile à justifier pour ces heures, mais aussi il y a d'autres raisons simulées, notamment la raison politico-sécuritaire. En plus, depuis l'entrée en vigueur du couvre-feu en RDC, il s'est constaté une sorte des violations des droits de l'homme à

* Chef de Travaux, Doctorant à la Faculté de Droit de l'Université de Lubumbashi et Avocat au Barreaudu Haut-Katanga, adressee-mail : martialkakudji@yahoo.fr

** Licencié en Droit à l'Université de Lubumbashi et chercheur. Courriel : mfuambaisidore@gmail.com

1 La mesure du couvre-feu a été levée dans certaines provinces où le coronavirus est à faible circulation. Elle est maintenue dans les autres provinces notamment Kinshasa, Lubumbashi etc., mais raccourcie de 22 heures à 4 heures. Lire Radio OKAPI, RDC-couvre-feu levé dans certaines provinces et raccourci à Kinshasa », publié le 14/04/2021 sur https://www.radiookapi.net/2021/04/1 4/actualite/societé/rdc-couvre-feu-leve-dans-certaines-provinces-et-racourci-kinshasa consulté le 21 avril 2021.

2 Luna Gay-Padooan, « Covid-19 en RDC: le premier couvre-feu national entre en vigueur la nuit », TV5MONDE, 20 décembre 2020, Permalien: https:/information.tv5monde.com/video/covid-19-en-r dc-le-premier-couvre-feu-national-entre-en-vigueur-la-nuit consulté le 15 mars 2021. 
Lubumbashi causées par la violence ${ }^{3}$ des services de sécurité qui œuvrent la nuit. Nous avons eu ces informations grâce aux interviews, témoignages et observations directes faites dans la ville de Lubumbashi.

Cela suscite quelques interrogations notamment: pourquoi s'acharner sur une personne pendant ces heures alors qu'il s'agit d'un couvre-feu sanitaire? Doit- on dire que la propagation de la Covid-19 est possible seulement la nuit? Pourquoi la non-observation des mesures barrières qui accompagnent ce couvre-feu, ne sont pas réprimées pendant la journée dans la ville? Ne doit-on pas conclure que les vraies raisons de l'instauration de ce couvre-feu sont simulées et ne sont pas sanitaires?

Cette réflexion analyse et critique en même temps, le couvre-feu selon la recherche faite dans la ville de Lubumbashi. Pour ce faire, nous avons structuré l'étude en trois points : d'abord, nous analysons le rôle qu'il joue réellement sur la propagation de la pandémie de Covid-19 (A); son impact sur le plan sécuritaire (B) et nous critiquons son impact négatif pour le respect des droits de l'homme pendant la nuit (C).

\section{A. Couvre-feu et la Covid-19 à Lubumbashi}

Dans ce point de notre réflexion, d'abord, nous définissons le concept 'couvre-feu' et celui de 'Covid-19' (I), nous parlons ensuite du régime juridique du couvre-feu et la compétence constitutionnelle de son instauration (II) et enfin, nous présentons le contexte de la prise de mesure de couvre-feu par le gouvernement congolais (III).

\section{Analyse lexicale}

Les deux concepts méritent une définition dans le cadre de cette étude, car ils sont constitutifs de notre sujet. C'est le Couvre-feu (1) et la Covid-19 (2).

\section{Couvre-feu}

Le concept 'couvre-feu' de manière triviale est une interdiction de sortir après une heure fixée.

Il est donc à définir comme une interdiction à la population de sortir de chez soi à partir d'une certaine heure, décrétée par une autorité publique. Cette heure est généralement la nuit et tôt matin. Cette interdiction peut être appliquée en temps de guerre ou de paix, par le gouvernement ou tout responsable d'un pays, d'une région ou d'une ville, pour des

3 Renato Galliano, «RDC: hausse des violences durant le couvre-feu », TV5MONDE, 21 janvier 2021, Permalien: https://information.tv5monde.com/video/rdc-hausse-des-violences-durant-le-couvr e-feu consulté le 15 mars 2021. 
raisons diverses, notamment lors de la déclaration d'une loi martiale ou de l'état de siège, soit encore pour une crise sanitaire ${ }^{4}$.

\section{Covid-19}

La Covid-19 (2019-nCoV) est une pandémie causée par le Coronavirus 2019. Elle est une maladie respiratoire aiguë qui touche principalement les poumons et elle a été identifiée pour la première fois en Chine plus précisément dans la province du Hubei, ville de Wuhan.

L'Organisation Mondiale de la Santé a déjà publié plusieurs documents en rapport avec les caractéristiques, symptômes et mode de transmission de la maladie de Covid-19. Mais, dans le cadre de cette réflexion, nous n'allons pas beaucoup entrer en détail.

Le point suivant cherche à identifier les règles de droit en vigueur en République Démocratique du Congo, applicable en matière de couvre-feu.

\section{Régime juridique du couvre-feu et la compétence constitutionnelle de son instauration en $R D C$}

Avant de voir les mesures qui ressortent de ce couvre-feu sanitaire, il est important de savoir le régime juridique de couvre-feu en République Démocratique du Congo et la compétence constitutionnelle de son instauration par une autorité gouvernementale.

Dans le vocabulaire de Cornu, on entend par 'régime' un système de règles, considéré comme un tout, soit en tant qu'il regroupe l'ensemble des règles relatives à une matière, soit en raison de la finalité à laquelle sont ordonnées les règles. C'est aussi un corps cohérent de règles ${ }^{5}$. Ainsi, chercher à savoir le régime juridique en matière de couvre-feu, consiste à réunir les règles de droit qui organisent ou qui règlementent cette matière.

Patient BAKADIKU estime que l'Etat en tant que personne morale de droit public vise dans sa mission de l'Etat gendarme, à garantir l'ordre social ${ }^{6}$. L'ordre social ou public en soi, est constitué de trois éléments classiques, notamment : la sécurité, la tranquillité (paix) et la salubrité publiques ${ }^{7}$. Mais, la liste a tendance de s'élargir avec l'ajout des autres éléments constitutifs de l'ordre public que la doctrine appelle "les éléments immatériels",

4 Pour approfondir sur le concept voir Permalien: https://fr.m.wikipedia.org/wiki/Couvre-feu consulté le 20 mars 2021.

5 Gérard Cornu, Vocabulaire juridique, 12e édition mise à jour « Quadrige », janvier 2018, p. 1855.

6 Patient BAKADIKU, "Impact de la mesure de couvre-feu sur les droits et libertés fondamentaux des citoyens en RDC », in Leganews.cd, publié le 17 décembre 2020, Permalien: https://www.legan ews.cd/index.php/actualites/institutions/2212-impact-de-la-mesure-de-couvre-feu-sur-les-droits-et-li bertes-fondamentaux-des-citoyens-en-rdc consulté le 21/04/2021.

7 Lire à ce sujet Vigny Landry Amoussou. L'ordre public sanitaire en Afrique francophone. Droit. Université de Bordeaux, 2017. Français. NNT: 2017BORD0662. tel-01814219, p. 25. 
entre autre : la moralité publique ${ }^{8}$; la vie et la dignité humaine, les valeurs essentielles de la société .

L'auteur précité ajoute que suivant la logique d'assurer la salubrité publique, l'Etat congolais représenté en l'espèce par le Président de la République a décrété le couvre-feu au motif de lutter contre la pandémie à corona virus constant la recrudescence à grande échelle des cas testés positifs à ladite pandémie, laquelle recrudescence constitue ici un trouble à l'ordre public. Ainsi, en décrétant ce couvre-feu, l'Etat espère une limitation de la propagation de cette pandémie sur le sol congolais ${ }^{10}$.

Si l'on peut considérer cette mesure comme prise dans le cadre du maintien de l'ordre public, nous estimons que la nature juridique de ce couvre-feu sanitaire est une police administrative ${ }^{11}$, car la salubrité en tant que l'un des objectifs de la police administrative qui exige un état sanitaire satisfaisant et se traduit par des mesures relatives à l'hygiène des personnes, des animaux et des choses et par la lutte contre la pollution (prévention des épidémies et épizooties, contrôle des comestibles exposés en vente, etc. $)^{12}$ 》.

Quid du titulaire du pouvoir de police en Droit congolais?

Interrogeant le Droit congolais, la compétence de décréter un couvre-feu n'est pas explicitement prévue par la Constitution. Néanmoins, il convient de relever que le couvrefeu, étant donné qu'il s'agit d'une mesure administrative, il relève de la compétence des autorités administratives. Or, aux termes de l'article 91 de la Constitution du 18 février 2006 , l'administration relève de la compétence exclusive du gouvernement ${ }^{13}$, dirigé selon le régime politique congolais par le Premier ministre ${ }^{14}$.

8 Il convient de noter que le Conseil d'État français a élargi la trilogie traditionnelle à des éléments relevant de ce qu'il dénomme la moralité publique. Sans faire sienne la conception rétrograde et dangereuse pour les libertés de «l'ordre moral » tel que le concevait notamment le régime de Vichy, il a néanmoins considéré comme licites l'interdiction de certains combats de boxe qui avaient "un caractère brutal et parfois sauvage ", ou d'affichages publicitaires en faveur de «messageries roses "; et, plus récemment, il a inclus dans la notion d'ordre public celle du respect obligatoire de la personne humaine. André Legrand et Céline Wiener, Le droit public. Droit constitutionnel, Droit administratif, Finances publiques, Institutions européennes, Édition Direction de l'information légale et administrative, Paris, 2017, pp. 116-118.

9 Jean-Claude Marin, "Propos introductifs", in Colloque "L'ordre public: regards croisés du Conseil d'Etat et de la Cour de cassation", Vendredi 24 février 2017, p. 3.

10 Patient BAKADIKU, art cit.

11 On définit la police administrative comme étant un « ensemble des moyens juridiques et matériels - réglementations, autorisations, défenses, injonctions, coercitions - mis en œuvre par les autorités administratives compétentes en vue d'assurer, de maintenir ou de rétablir l'ordre public; on distingue la police administrative générale chargée du maintien de la sécurité, de la tranquillité et de la salubrité publiques, des polices administratives spéciales applicables à telle ou telle activité, par ex. chemin de fer... ». Gérard Cornu, op cit., p. 1635.

12 Gérard Cornu, op cit., p. 1988.

13 « Le Gouvernement dispose de l'administration publique, des Forces armées, de la Police nationale et des services de sécurité ». Article 91 de la Constitution du 12 février 2006.

14 « [Le gouvernement] est dirigé par le Premier ministre, chef du Gouvernement ». Article 90, idem. 
En Droit comparé (français) les titulaires du pouvoir de police administrative existent presque autant que de niveaux territoriaux d'administration. Les préfets ont été investis de ce pouvoir dans leur département pendant la Révolution et les maires l'exercent dans leur commune depuis la loi de 1884, reprise dans le Code général des collectivités territoriales. Par ailleurs, le Conseil d'État a admis en 1919 que le président de la République dispose du même pouvoir au niveau national (depuis la Constitution de 1946, ses compétences en la matière ont été transférées au chef du gouvernement) $)^{15}$.

Nous estimons que la compétence de décréter la mesure de couvre-feu en République Démocratique du Congo varie selon l'étendue du territoire concerné. Si c'est un couvre-feu national, le pouvoir de police au niveau national revient au Chef du gouvernement (le Premier ministre) et ce, sur base de l'article 91 de la Constitution qui veut que le « le Gouvernement dispose de l'administration publique », couvre-feu sanitaire étant une mesure purement administrative (voir supra). Quant au Président de la République, l'on peut tirer le fondement de cette compétence de la pratique, considérant qu'il lui revient en dehors de toute délégation législative et en vertu de ses pouvoirs propres, de déterminer des mesures de police qui doivent en tout état de cause être appliquées dans l'ensemble du territoire, comme la jurisprudence française le note avec l'arrêt Labonne du 8 août $1919^{16}$.

Mais, lorsqu'il s'agit d'une province c'est le gouverneur de province, lorsqu'il s'agit d'une entité territoriale décentralisée (ETD) ${ }^{17}$ Ce sont les autorités respectives de chacune de ces entités qui doivent prendre de mesure de police.

\section{Contexte de la prise de mesure de couvre-feu par le gouvernement congolais}

En effet, dans le cadre de la lutte contre la propagation de la pandémie de Covid-19, le Comité multisectoriel de lutte contre la pandémie du coronavirus présidé par le président de la République, Felix Antoine Tshisekedi a décrété un couvre-feu le vendredi 18 décembre

15 André Legrand et Céline Wiener, Le droit public. op cit., p. 117.

16 Un décret de 1899, ancêtre du Code de la route français, avait rendu obligatoire pour tous les conducteurs d'automobiles un « certificat de capacité », qui pouvait leur être retiré par le préfet en cas d'infraction grave aux règles de circulation. Saisi par un contrevenant auquel cette mésaventure était arrivée, le Conseil d'État déclara le décret légal au motif « qu'il appartient au chef de l'État, en dehors de toute délégation législative et en vertu de ses pouvoirs propres, de déterminer celles des mesures de police qui doivent en tout état de cause être appliquées dans l'ensemble du territoire ». Ibidem.

17 Aux termes de l'article 3 de la Constitution « Les provinces et les entités territoriales décentralisées de la République Démocratique du Congo sont dotées de la personnalité juridique et sont gérées par les organes locaux. Ces entités territoriales décentralisées sont la ville, la commune, le secteur et la chefferie ». Constitution de la République Démocratique du Congo modifiée par la Loi $\mathrm{n}^{\circ} 11 / 002$ du 20 janvier 2011 portant révision de certains articles de la Constitution de la République Démocratique du Congo du 18 février 2006. 
2020; il s'agit d'un couvre-feu sanitaire et général ${ }^{18}$. L'annonce a été faite au cours de la réunion tenue dans la soirée du 15 décembre au Palais de la Nation ${ }^{19}$.

D'après la version officielle, la raison majeure et apparente de l'instauration de ce couvre-feu est de « lutter contre la propagation du Coronavirus dont le nombre de cas est reparti à la hausse depuis le mois de novembre ${ }^{20}$ »)

Le Gouvernement congolais avait voulu enrayer la transmission du Covid-19, qui touchait surtout la capitale Kinshasa ${ }^{21}$. Selon eux, le pays avait connu une augmentation du nombre de nouveaux cas de contamination depuis le début du mois de décembre, avec une moyenne de 100 nouveaux cas par jour dans la capitale, Kinshasa, l'épicentre de la pandémie $^{22}$.

C'est dans ce cadre qu'il avait annoncé qu'un couvre-feu sera imposé de 21 heures à 5 heures après un pic dans les cas de Coronavirus et la restriction avait débuté vendredi. Le ministre congolais de la communication, Julino Makelele, avait annoncé cette mesure parmi d'autres, notamment la fermeture anticipée des écoles pour les vacances de Noel, l'interdiction des cérémonies avant les enterrements et l'interdiction des rassemblements publics de plus de 10 personnes ${ }^{23}$. Le ministère de la santé a annoncé mercredi qu'un nombre record de 333 personnes avaient été testées positives la veuille ${ }^{24}$.

La véracité de cette raison est difficile à démontrer d'autant plus que les mesures barrières restées en application après la levée d'état d'urgence sanitaire par le Président de la République, étaient à mesure de lutter contre la propagation du virus. D'ailleurs ces mesures semblent tomber en désuétude parce que nous constatons leur non-respect même dans des milieux publics, comme dans les moyens de transport ${ }^{25}$ où il $\mathrm{y}$ a des fortes probabilités de contamination rapide de la pandémie.

18 On note que le couvre-feu peut-être général (concernant toute la population sans discrimination aucune), ou se limitant à une catégorie de la population bien déterminée (les enfants, les femmes, etc.). Patient BAKADIKU, art cit.

19 Albert Muanda, « Covid-19 : couvre-feu et vacances anticipées des écoliers dès ce vendredi 18 décembre, sur toute l'étendue du territoire national ", in Environews-rdc.org., publié le 16 décembre 2020, Permalien : https://www.environews.rdc.org/2020/12/16/covid-19-couvre-feu-et-v acances-anticipees-des-ecoliers-des-ce-vendredi-18-decembre-sur-toute-letendue-du-territoire-nati onal/ Consulté le 21/04/2021.

20 Luna Gay-Padooan, art. cit.

21 Permalien : https://m.lavenir.net/cnt/dmf20201219-01539034/coronavirus-debut-du-couvre-feu-de -21h-a-5h-en-rdc consulté 22 mars 2021.

22 Permalien: https://www.bbc.com/afrique/region-55333392 consulté le 15 mars 2021.

23 Permalien: https://www.bbc.com/afrique/region-55333392 consulté le 15 mars 2021.

24 Ibidem.

25 Pourtant il est exigé un respect strict des gestes barrières dans tous les lieux publics et dans les transports publics et privés, notamment dans les véhicules, les trains, les avions et les motos. Chambre de Commerce et d'Industrie Franco-Congolaise (CCI), " Kinshasa-Mise en place d'un couvre-feu à compter du vendredi 18 décembre 2020 », publié le 17/12/2020, en ligne, Permalien : https://www.ccife-rdcongo.org/actualites/n/news/kinshasa-mise-en-place-dun-couvre-feu-a-compt er-du-vendredi-18-decembre-2020.html. 
La décision du couvre-feu a été consécutive à celle ordonnant l'application stricte des gestes barrières (port correct et obligatoire des masques, le respect de la distanciation physique, le lavage régulier des mains et la prise de température $)^{26}$, lesquelles souffrent d'inapplication quasi-totale dans la ville de Lubumbashi, parce que même les éléments de la police censés contrôler leur mise en œuvre ne sont pas de maitres exemplaires, c'est-à-dire ils marchent sans porter les masques, c'est difficile que eux même arrêtent une personne qui n'en porte pas.

Le gouvernement a également interdit les marches publiques, les productions artistiques et les kermesses, ainsi que les cérémonies festives et les réunions de plus de dix personnes. Ces mesures ont été annoncées un jour après le discours du chef de l'Etat Felix Tshisekedi devant les deux chambres du Parlement. Le Chef de l'Etat a invité la population congolaise à "une vigilance tous azimuts vis-à-vis de la deuxième vague de Covid-19 en RDC, en particulier à Kinshasa, où l'on enregistrait plusieurs cas importés de Covid-19”. En prenant de telles dispositions, le gouvernement entendait éviter la propagation de la deuxième vague de Covid-19 en République Démocratique du Congo ${ }^{27}$.

Pendant le couvre-feu, en principe la circulation est interdite pour toute personne. Exceptionnellement, des personnes munies de l'autorisation spéciale de l'autorité provinciale sont permises de circuler. Cette autorisation n'est pas exigée s'il y a une urgence sanitaire ${ }^{28}$.

Par ailleurs, il est à noter que le but ultime d'instauration d'un couvre-feu est de permettre aux forces de l'ordre, civiles ou militaires, de mieux assurer la sécurité de la zone sous couvre-feu, de limiter la libre circulation d'une certaine catégorie de personnes, comme les femmes ou les mineurs ou de protéger la population d'un danger ${ }^{29}$. Suivant cette logique, nous trouvons injustifié un couvre-feu sanitaire, pris dans le but de lutter contre la propagation de la maladie contagieuse allant de 21 heures à 5 heures du matin alors que pendant la journée les personnes circulent librement sans observer les gestes barrières définis par le gouvernement.

Mais, une telle mesure pendant la nuit peut satisfaire à ce but seulement pour les activités nocturnes, notamment les bars ou les boites de nuit ${ }^{30}$, les fêtes etc., or, ces activités étaient déjà interdites dans la série des gestes barrières. Bien que ce soit la nuit que les bars fonctionnent et que certaines fêtes s'organisent, la possibilité de propager la maladie de Covide-19 nous semble mineur pendant la nuit.

Nous sommes de même avis avec Patient BAKADIKU qui pense que : « qu'il s'observe l'absence de lien rationnel entre l'objectif recherché par l'Etat et le moyen utilisé. De

26 Permalien: https://www.bbc.com/afrique/region-55333392 consulté le 15 mars 2021.

27 Ibidem.

28 Chambre de Commerce et d'Industrie Franco-Congolaise (CCI), art cit.

29 Permalien: https://www.bbc.com/afrique/region-55333392 consulté le 15 mars 2021.

30 Il ressort des mesures qui accompagnent ce couvre-feu que le fonctionnement des églises et des débits de boissons avant le couvre-feu n'est pas interdit, mais avec strict respect des gestes barrières. Chambre de Commerce et d'Industrie Franco-Congolaise (CCI), art cit. 
plus, cette mesure de couvre-feu n'est pas proportionnelle car, impactant négativement les droits et libertés des individus ${ }^{31} \gg$.

De la sorte, l'argument qui consiste à dire qu'un tel couvre-feu a pour l'unique finalité la lutte contre la pandémie de Covid-19, n'emporte pas notre intime conviction, il doit y avoir des raisons simulées, c'est ce que le point suivant tente d'expliquer.

\section{B. Couvre-feu et la sécurité à Lubumbashi}

Outre la raison officielle du couvre-feu ci-haut, ce dernier a aussi d'impact sur le plan sécuritaire en RDC généralement et dans la ville de Lubumbashi plus particulièrement. Cet impact nous semble positif, en ce qu'il constitue un moyen nécessaire pour le renforcement de la sécurité. En effet, le couvre-feu a instauré une sorte de discipline dans le chef de la population congolaise du fait qu'avant l'on pouvait se déplacer n'importe quelle heure sans problème, et c'est dans ce mouvement que les groupes des malfrats se déplaçaient sans contrôle de services de sécurités.

Cette observation faite dans la ville de Lubumbashi est vérifiable dans la mesure où, le taux de criminalité nocturne pendant ces trois mois est insignifiant. Certains quartiers de cette ville, dans lesquels l'on décriait l'insécurité nocturne très souvent, comme quartiers Kassapa et Gambela qui étaient dans le noir (sans énergie électrique) depuis la fin du mois de mars ${ }^{32}$ jusqu'au 14 avril, mais le taux d'insécurité était insignifiant. Pourtant le manque d'énergie électrique est une cause criminogène par excellence. Les groupes de malfrats se servent de l'obscurité pour bien opérer pendant la nuit.

Ce climat de tranquillité et sécurité sociales dans ces quartiers pendant cette période de crise d'électricité est dû à la présence des éléments de sécurités (patrouilleurs) dans la route pendant la nuit pour raison de patrouille. Mais, la présence de ces derniers en route pendant la nuit inquiète la population. Il y a certains d'entre eux, qui s'évertuent de violenter les personnes avant même qu'il soit 21 heures (le début du couvre-feu). Les habitants de Lubumbashi ont même dénoncé une hausse de l'insécurité chronique avec le couvre-feu décrété le 18 décembre pour faire face au Coronavirus ${ }^{33}$. Cette insécurité n'est pas causé par les voleurs dans les maisons habitées pendant la nuit comme c'était le cas les mois antérieurs, plutôt c'est une insécurité dans la route quand il commence à faire sombre. Il s'agit là d'un autre aspect que nous expliquons dans le point suivant de notre analyse.

31 Patient BAKADIKU, art cit.

32 Cette maque d'électricité a débuté la nuit du mardi 30 mars et il y a de cela deux semaines avec quelques jours, la cause de cette panne selon la Direction régionale du sud est le vol de 6 pylônes et les câbles électriques en cuivre nu. Pour en savoir plus lire Adrien Ambanengo, "RDC: Une partie de la ville de Lubumbashi plongée dans le noir», 31 mars 2021, consulté le 13 avril 2021 à 08 heures. Permalien : https://www.politico.cd/encontinu/2021/03/31/rdc-une-partie-de-la-ville-de -lubumbashi-plongee-dans-le-noir.html/80608.

33 Renato Galliano, «RDC: hausse des violences durant le couvre-feu », art. cit. 


\section{Couvre-feu et les droits de l'homme à Lubumbashi}

Outre les avantages que le couvre-feu présente aujourd'hui dans la ville de Lubumbashi (même dans d'autres villes des provinces plus peuplées du pays), cette mesure a d'impact négatif sur le plan du respect des droits de l'homme, car depuis son instauration il a constitué une porte ouverte aux violations des droits de l'homme causées par la violence ${ }^{34}$ des services de sécurité qui œuvrent la nuit. Nous ne parlons pas ici des violations des droits dans le sens de restrictions de certaines libertés garanties tant par la Constitution et textes législatifs de la République Démocratique du Congo et les instruments juridiques internationaux relatifs aux droits de l'homme, telles que les libertés de circulation, d'aller et de revenir, de réunions pacifiques et d'association, de cultes etc. ${ }^{35} \mathrm{Car}$, ces libertés peuvent être connaitre de restriction proportionnées dans le cadre de la préservation de la population d'un mal quelconque. C'est-à-dire une restriction qui profite à la population bénéficiaire des droits et libertés limités. Plutôt notre recherche se base sur les violations des droits de l'homme causées par les agents de services de sécurité qui font la patrouille.

En effet, vu que les heures du déplacement sont devenues règlementer, depuis son instauration, dès qu'il fait 19 heures (parfois avant qu'il soit ces heures), dans la ville nous constatons que toute personne se hâte de retourner chez soi (à la maison), ce qui crée plus souvent les embouteillages, la hausse du prix de transport et aussi des accidents de circulation. Ces embouteillages et risques que nous constatons, suivant la théorie de 'cause à effet' sont dus suite aux abus que les services de sécurité, notamment la police et l'armée, qui œuvrent pendant la nuit, commettent presque chaque jour.

En effet, les heures règlementées sont 21 heures à 5 heures, repoussées naguère de 22 heures à 4 heures, il arrive que les militaires et les policiers dès qu'il fait seulement noir dans la ville, avant ces heures (c'est-à-dire 19 heures 30 à 20 heures passées) traquassent la population, sans pitié ni tolérance.

Pour des amples exemples, dans la commune de Lubumbashi, le quartier Gambela proche de l'arrêt Texaco, il y a une bande des militaires qui se nourrissent des motards et passagers avant qu'il soit 21 heures. Il en est ainsi, parfois plus pire dans d'autres quartiers de la ville de Lubumbashi ${ }^{36}$. Dès qu'ils arrêtent une personne ou un motard, ces éléments de sécurité en uniforme, la première des choses à faire c'est ravir le téléphone et l'argent et laisser la personne partir. Malheureux sera celui qui n'a ni l'argent ni téléphone, car dans ce moment-là, il peut subir même des atteintes physiques. Alors que la Constitution de la République Démocratique du Congo exige que toute personne arrêtée soit immédiatement informée des motifs de son arrestation et de toute accusation portée contre elle et ce, dans la langue qu'elle comprend. Et qu'elle soit immédiatement informée de ses droits ${ }^{37}$. Mais

34 Renato Galliano, «RDC: hausse des violences durant le couvre-feu », art. cit.

35 Pour cet aspect voir Patient BAKADIKU, art cit.

36 Le même phénomène se déroule du coté Kassapa, entre le cités universitaires et le camp blanc et rouge.

37 Article 18 de la Constitution du 18 février 2006. 
les services de sécurité arrêtent et détiennent les personnes sous prétexte de violation de mesure de couvre-feu, lorsqu'on vérifie l'heure d'arrestation, pour la plus part de cas, c'est avant les heures couvertes par la mesure.

La Constitution exige aussi que tout détenu puisse bénéficier d'un traitement qui préserve sa vie, sa santé physique et mentale ainsi que sa dignité ${ }^{38}$. Plus pire, durant les premiers jours du couvre-feu, certaines personnes qui se sont fait arrêter la nuit (bien que déjà à 21 heures) étaient mises dans les cachots et fouettées par les policiers et les images ont circulé dans nos téléphones.

La même pratique continue dans le quartier Kigoma avec les militaires qu'on appelle communément 'Commandos' qui frappent les contrevenants à cette mesure de couvre-feu avec les morceaux de planche. C'est de la torture pourtant, un tel comportement constitue une violation de l'article 61 de la Constitution de la République Démocratique du Congo qui dispose « [e]n aucun cas, et même lorsque l'état de siège ou l'état d'urgence aura été proclamé conformément aux articles 85 et 86 de la présente Constitution, il ne peut être dérogé aux droits et principes fondamentaux énumérés ci-après : le droit à la vie; l'interdiction de la torture et des peines ou traitements cruels, inhumains ou dégradants $»{ }^{39}$.

Ces violences constituent le non-respect de la dignité de la personne humaine qui ne doit souffrir d'aucune atteinte même pendant les mesures restrictives aux droits et libertés de personne, notamment l'état de siège ou l'état d'urgence, le couvre-feu etc.

Les conséquences de leurs abus sont majeures, notamment : les embouteillages; les accidents de circulations routières; les risques de mort causées par les inondations lorsqu'il y a forte pluie vespérale comme celle du 26 mars qui a causé huit morts dans le quartier Kigoma $^{40}$; les vols et extorsion; les coups et blessures etc. Donc, il revient aux autorités de la ville de veiller à la quiétude de la population pendant cette période de couvre-feu.

\section{Conclusion}

En conclusion, le couvre-feu sanitaire décrété en République Démocratique du Congo est une mesure efficace pour la gestion de problème sécuritaire dans la ville de Lubumbashi particulièrement, dans la mesure où il permet le contrôle de mouvement de la population pendant les heures où les délinquants opèrent plus facilement (la nuit). Mais, celui-ci n'est pas à mesure de lutter contre la propagation du virus responsable de Covid-19, car la nuit ne constitue pas un moment propice pour sa propagation. C'est en revanche pendant la journée qu'il peut se propager avec facilité. Mais, cette expérience du couvre-feu démontre que le la règlementation des heures de sorties peut aider le gouvernement de la République

38 Ibidem.

39 Article 61, Idem.

40 Le soir du 26 mars il y a eu dans la ville de Lubumbashi une forte pluie, il y avait d'inondations presque dans toutes les grandes routes. Terrifiées par le comportement biaisé des éléments de sécurité, certaines personnes ont dû marcher avec cette pluie, les eaux ont emporté plus de huit personnes dont les corps ont été retrouvés sans vie. 
Démocratique du Congo de bien gérer l'insécurité, l'un de défis majeurs que connait cet Etat.

Ainsi, nous proposons au gouvernement de veiller au respect et à la mise en œuvre des gestes barrières, qui d'ailleurs à ce jour semblent être tombées en désuétude car personne ne les respectent. Et aussi, de veiller à ce que les services de sécurité qui patrouillent les heures couvertes (21 heures à 05 heures et plus récemment ramenées de 22 heures à 04 heures) ne s'en servent du couvre-feu pour violenter la population.

\section{Bibliographie}

1. André Legrand et Céline Wiener, Le droit public. Droit constitutionnel, Droit administratif, Finances publiques, Institutions européennes, Édition Direction de l'information légale et administrative, Paris, 2017.

2. Adrien Ambanengo, «RDC: Une partie de la ville de Lubumbashi plongée dans le noir », 31 mars 2021, consulté le 13 avril 2021 à 08 heures. Permalien : https://www.p olitico.cd/encontinu/2021/03/31/rdc-une-partie-de-la-ville-de-lubumbashi-plongee-da ns-le-noir.html/80608.

3. Albert Muanda, " Covid-19 : couvre-feu et vacances anticipées des écoliers dès ce vendredi 18 décembre, sur toute l'étendue du territoire national », in Environewsrdc.org., publié le 16 décembre 2020, Permalien : https://www.environews.rdc.org/20 20/12/16/covid-19-couvre-feu-et-vacances-anticipees-des-ecoliers-des-ce-vendredi-18 -decembre-sur-toute-letendue-du-territoire-national/

4. Chambre de Commerce et d'Industrie Franco-Congolaise (CCI), « Kinshasa-Mise en place d'un couvre-feu à compter du vendredi 18 décembre 2020 », publié le 17/12/2020, en ligne, Permalien : https://www.ccife-rdcongo.org/actualites/n/news/kin shasa-mise-en-place-dun-couvre-feu-a-compter-du-vendredi-18-decembre-2020.html

5. Constitution de la République Démocratique du Congo modifiée par la Loi $\mathrm{n}^{\circ} 11 / 002$ du 20 janvier 2011 portant révision de certains articles de la Constitution de la République Démocratique du Congo du 18 février 2006.

6. Gérard Cornu, Vocabulaire juridique, 12e édition mise à jour « Quadrige », janvier 2018.

7. https://fr.m.wikipedia.org/wiki/Couvre-feu.

8. https://m.lavenir.net/cnt/dmf20201219-01539034/coronavirus-debut-du-couvre-feu-de -21h-a-5h-en-rdc.

9. https://www.bbc.com/afrique/region-55333392.

10. Jean-Claude Marin, "Propos introductifs », in Colloque "L'ordre public: regards croisés du Conseil d'Etat et de la Cour de cassation", Vendredi 24 février 2017.

11. Luna Gay-Padooan, "Covid-19 en RDC: le premier couvre-feu national entre en vigueur la nuit », in TV5MONDE, 20 décembre 2020, Permalien: https://information.t v5monde.com/video/covid-19-en-rdc-le-premier-couvre-feu-national-entre-en-vigueur -la-nuit. 
12. Patient BAKADIKU, "Impact de la mesure de couvre-feu sur les droits et libertés fondamentaux des citoyens en RDC », in Leganews.cd, publié le 17 décembre 2020, Permalien : https://www.leganews.cd/index.php/actualites/institutions/2212-impact-d e-la-mesure-de-couvre-feu-sur-les-droits-et-libertes-fondamentaux-des-citoyens-en-r dc.

13. Radio OKAPI, RDC-couvre-feu levé dans certaines provinces et raccourci à Kinshasa», publié le 14/04/2021 sur https://www.radiookapi.net/2021/04/14/actualite/societé /rdc-couvre-feu-leve-dans-certaines-provinces-et-racourci-kinshasa.

14. Renato Galliano, "RDC: hausse des violences durant le couvre-feu », IN TV5MONDE, 21 janvier 2021, Permalien: https://information.tv5monde.com/video/rdc-ha usse-des-violences-durant-le-couvre-feu.

15. Vigny Landry Amoussou. L'ordre public sanitaire en Afrique francophone. Droit. Université de Bordeaux, 2017. Français. NNT: 2017BORD0662. tel-01814219. 